\title{
Prehospital fibrinolysis followed by urgent percutaneous coronary intervention after ST-elevation myocardial infarction
}

\author{
Umair Khalid*,1, Hani Jneid ${ }^{1}$ \& Ali Emin Denktas ${ }^{1}$ \\ ${ }^{1}$ Baylor College of Medicine, Houston, TX 77030, USA \\ *Author for correspondence: Tel.: +1 713798 0284; Fax: +1 713798 0277; mukhalid@bcm.edu
}

'The primary goal of emergent reperfusion in patients with ST-elevation myocardial infarction (STEMI) is to reduce the total ischemic time (TIT); for STEMI patients in whom there is an expected delay in primary $\mathrm{PCl}$, we suggest the use of half-dose fibrinolytic therapy, followed by urgent transfer for coronary angiography without the routine use of glycoprotein IIB/IIIA inhibitors, as long as the patient is adequately loaded with a P2 $\mathrm{Y}_{12}$ inhibitor and aspirin"”

First draft submitted: 26 January 2018; Accepted for publication: 14 March 2018; Published online: 16 May 2018

Keywords: fibrinolysis • infarction • percutaneous coronary intervention • STEMI

The primary goal of emergent reperfusion in patients with ST-elevation myocardial infarction (STEMI) is to reduce the total ischemic time (TIT); which encompasses all events from the time of culprit vessel occlusion to coronary blood flow restoration by either balloon angioplasty in cardiac catheterization lab or fibrinolysis. Overwhelming data support the direct association of TIT with cardiovascular outcomes, ranging from animal experiments of coronary artery ligation [1] and magnetic resonance imaging of infarct zones [2] to large clinical studies and meta-analyses [3]. An analysis of over 1700 STEMI patients showed that 1-year mortality increased by $7.5 \%$ for each 30 -min delay in reperfusion [4]. The 2013 ACCF/AHA guidelines for the management of STEMI [5] and the 2017 AHA/ACC Clinical Performance and Quality Measures for AMI [6] emphasize several metrics of timeliness of reperfusions including: the first medical contact-device time (in PCI-capable hospitals), first medical contact-device time (in transferred patients), door-to-needle time and door-in-door-out time. All of these measures are in fact components of TIT. However, while these metrics are readily measurable and reproducible, the TIT is subject to recollection bias and estimation errors and is difficult to measure.

One approach to decrease the TIT in STEMI patients who need transfer to a PCI capable facility or anticipate any delay in PCI, is to administer fibrinolysis followed by urgent PCI [5]. Excluding data from the angioplasty era, there are six major trials which studied fibrinolysis followed by urgent PCI [7-12]. The control group for three of these studies $[7,8,12]$ was primary PCI only, while for the rest [9-11], it was fibrinolysis plus conventional strategy (the latter strategy mandated STEMI patients to be transferred for invasive management within 3-24 h as subsequently adopted in the STEMI guidelines). Except for the Assessment of the Safety and Efficacy of a New Treatment Strategy with Percutaneous Coronary Intervention (ASCENT)- 4 trial [7], all studies reported a reduction in the primary efficacy outcome in the 'fibrinolysis plus PCI' group (statistically significant reduction in three studies $[8,9,11]$, and nonsignificant trend in two studies $[10,12])$. In the ASCENT-4 trial [7], the primary composite outcome was largely driven by recurrent $\mathrm{MI}$, and patients in the 'fibrinolysis plus PCI' group had a significantly lower clopidogrel use compared with primary PCI group. Notably, a fourth of STEMI patients [13] can still have TIMI 0 flow after fibrinolysis with tenecteplase. This may explain why fibrinolysis followed by PCI is linked to better outcomes compared with fibrinolysis followed by conservative strategy [9-11].

In all the aforementioned trials, the safety end points were also assessed [7-12]. There were no significant differences in either major bleeding or intracranial bleeding in the three trials that compared fibrinolytic therapy followed by urgent PCI with conventional strategy [9-11]. However, in two other trials [7,12], there was a signal toward increased bleeding in the fibrinolytic therapy plus urgent PCI group, when compared with primary PCI. The AMICO trial 
evaluated half-dose fibrinolytic dose without a mandated IIB/IIIA inhibitor use [8], comparing with primary PCI. The primary outcome, 1-month mortality, as well as all secondary outcomes including combined triple end point of death, reinfarction, or stroke, infarct-related artery TIMI flow grades and incidence of Killip class IV symptoms, were superior in the half-dose fibrinolytic therapy plus urgent PCI group when compared with primary PCI [8]. This was achieved without an increase in bleeding rate. When the infarct related artery patency is achieved before the patient arrives to the PCI hospital, the rate of cardiogenic shock events appears to be lower as well. This has been shown in a recent study of STEMI patients, where a strategy of half-dose reteplase followed by urgent primary PCI was associated with lower incidence of cardiogenic shock and reduced in-hospital mortality compared with primary PCI alone [14]. In another study of STEMI patients, none of the patients receiving half-dose reteplase and clopidogrel $600 \mathrm{mg}$ had intracranial bleeding [15].

The STREAM trial [16] had a slightly different design; it studied all STEMI patients who presented within $3 \mathrm{~h}$ after symptom onset and who were unable to undergo primary PCI within $1 \mathrm{~h}$. These patients were randomized to receive either (delayed) PCI or prehospital full-dose fibrinolysis (amended to half dose in patients $\geq 75$ years of age) before transport to a PCI-capable hospital. The primary outcome was mortality, shock, MI or CHF within 30 days, and there were no statistically significant differences between the groups. However, emergent coronary angiography was necessary in 36\% of patients in the fibrinolysis group patients. Rashid et al. [13] recently concluded that a fourth of all STEMI patients who received fibrinolysis have TIMI 0 flow at the time of follow-up coronary angiography. Both these studies show that while fibrinolytic therapy is crucial in reducing TIT and improving outcomes in STEMI patients with expected delay in primary PCI, significant fraction of patients still have inadequate restoration of coronary blood flow after fibrinolysis. Therefore, we advocate that these patients should be rushed to the nearest PCI capable facility for urgent coronary angiography regardless of their hemodynamic status and ongoing anginal or ischemic EKG changes. Any delay in STEMI patients increases the TIT and worsens clinical outcomes. The need to wait in the contemporary era of increasing use of radial interventions and safer anticoagulant regimen (e.g., bivalirudin) is debatable and should to be reassessed.

The latest AHA/ACC guidelines [5] recommend elective transfer for coronary angiography between 3 and $24 \mathrm{~h}$ after fibrinolysis. This may be due to concern for possible increased bleeding in fibrinolysis followed by urgent PCI. The signal for increased bleeding was seen in trials that used either full dose fibrinolysis, or half-dose fibrinolysis coupled with a glycoprotein IIB/IIIA inhibitor. We already know from the AMICO trial that there is no increased bleeding risk if half-dose fibrinolytic is used without a glycoprotein IIB/IIIA inhibitor while maintaining improved ischemic outcomes [8]. In fact, major bleeding was decreased in fibrinolysis plus urgent PCI compared with primary PCI group in this trial. It was hypothesized that due to improved ischemic outcomes, there was less need for aggressive measures such as use of intra-aortic balloon pump or reintervention, which may lead to increased bleeding. A meta-analysis also showed that fibrinolysis followed by urgent PCI had significantly improved ischemic outcomes without any increased bleeding risks compared with standard care [17].

\section{Conclusion}

In conclusion, for STEMI patients in whom there is an expected delay in primary PCI, we suggest the use of half-dose fibrinolytic therapy, followed by urgent transfer for coronary angiography without the routine use of glycoprotein IIB/IIIA inhibitors, as long as the patient is adequately loaded with a P2 $\mathrm{Y}_{12}$ inhibitor and aspirin. The addition of a glycoprotein IIB/IIIA inhibitor may be considered when patients are not adequately pretreated with dual antiplatelet therapy or as bail-out/rescue strategy.

Financial \& competing interests disclosure

The authors have no relevant affiliations or financial involvement with any organization or entity with a financial interest in or financial conflict with the subject matter or materials discussed in the manuscript. This includes employment, consultancies, honoraria, stock ownership or options, expert testimony, grants or patents received or pending, or royalties.

No writing assistance was utilized in the production of this manuscript.

\section{References}

1. Reimer KA, Lowe JE, Rasmussen MM, Jennings RB. The wave front phenomenon of ischemic cell death: myocardial infarct size vs. duration of coronary occlusion in dogs. Circulation 56, 786-794 (1977).

2. Eitel I, Desch S, Fuernau G et al. Prognostic significance and determinants of myocardial salvage assessed by cardiovascular magnetic resonance in acute reperfused myocardial infarction. J. Am. Coll. Cardiol. 55(22), 2470-2479 (2010). 
3. The European Myocardial Infarction Project Group. Prehospital thrombolytic therapy in patients with suspected acute myocardial infarction. N. Engl. J. Med. 329, 383-389 (1993).

4. Dudek D, Rakowski T, Dziewierz A, Mielecki W. Time delay in primary angioplasty: how relevant is it? Heart 93(10), 1164-1166 (2007).

5. Levine GN, Bates ER, Blankenship JC et al. 2015 ACC/AHA/SCAI focused update on primary percutaneous coronary intervention for patients with ST-elevation myocardial infarction: an update of the 2011 ACCF/AHA/SCAI guideline for percutaneous coronary intervention and the $2013 \mathrm{ACCF} / \mathrm{AHA}$ guideline for the management of st-elevation myocardial infarction. J. Am. Coll. Cardiol. 67(10), 1235-1250 (2016).

6. Jneid H, Addison D, Bhatt DL et al. 2017 AHA/ACC clinical performance and quality measures for adults with ST-elevation and non-ST-elevation myocardial infarction: a report of the American College of Cardiology/American Heart Association Task Force on Performance Measures. J. Am. Coll. Cardiol. 70(16), 2048-2090 (2017).

7. Assessment of the Safety and Efficacy of a New Treatment Strategy with Percutaneous Coronary Intervention (ASSENT-4 PCI) investigators. Primary versus tenecteplase-facilitated percutaneous coronary intervention in patients with ST-segment elevation acute myocardial infarction (ASSENT-4 PCI): randomized trial. Lancet 367(9510), 569-578 (2006).

8. Denktas AE, Athar H, Henry TD et al. Reduced-dose fibrinolytic acceleration of ST-segment elevation myocardial infarction treatment coupled with urgent percutaneous coronary intervention compared with primary percutaneous coronary intervention alone results of the AMICO (Alliance for Myocardial Infarction Care Optimization) registry. JACC Cardiovasc. Interv. 1(5), 504-510 (2008).

9. Cantor WJ, Fitchett D, Borgundvaag B et al. TRANSFER-AMI Trial Investigators. Routine early angioplasty after fibrinolysis for acute myocardial infarction. N. Engl. J. Med. 360(26), 2705-2718 (2009).

10. Bøhmer E, Hoffmann P, Abdelnoor M, Arnesen H, Halvorsen S. Efficacy and safety of immediate angioplasty versus ischemia-guided management after thrombolysis in acute myocardial infarction in areas with very long transfer distances results of the NORDISTEMI (NORwegian study on DIstrict treatment of ST-elevation myocardial infarction). J. Am. Coll. Cardiol. 55(2), 102-110 (2010).

11. Di Mario C, Dudek D, Piscione F et al. CARESS-in-AMI (Combined Abciximab RE-teplase Stent Study in Acute Myocardial Infarction) Investigators. Immediate angioplasty versus standard therapy with rescue angioplasty after thrombolysis in the Combined Abciximab REteplase Stent Study in Acute Myocardial Infarction (CARESS-in-AMI): an open, prospective, randomized, multicenter trial. Lancet 371(9612), 559-568 (2008).

12. Ellis SG, Tendera M, de Belder MA et al. FINESSE Investigators. Facilitated PCI in patients with ST-elevation myocardial infarction. $N$. Engl. J. Med. 358(21), 2205-2217 (2008).

13. Rashid MK, Guron N, Bernick J et al. Safety and efficacy of a pharmacoinvasive strategy in st-segment elevation myocardial infarction: a patient population study comparing a pharmacoinvasive strategy with a primary percutaneous coronary intervention strategy within a regional system. JACC Cardiovasc. Interv. 9(19), 2014-2020 (2016).

14. Bhatt NS, Solhpour A, Balan P et al. Comparison of in-hospital outcomes with low-dose fibrinolytic therapy followed by urgent percutaneous coronary intervention versus percutaneous coronary intervention alone for treatment of ST-elevation myocardial infarction. Am. J. Cardiol. 111(11), 1576-1579 (2013).

15. Solhpour A, Chang KW, Balan P et al. Comparison of outcomes for patients $\geq 75$ years of age treated with prehospital reduced-dose fibrinolysis followed by percutaneous coronary intervention versus percutaneous coronary intervention alone for treatment of ST-elevation myocardial infarction. Am. J. Cardiol. 113(1), 60-63 (2014).

16. Armstrong PW, Gershlick AH, Goldstein P et al. STREAM Investigative Team. Fibrinolysis or primary PCI in ST-segment elevation myocardial infarction. N. Engl. J. Med. 368(15), 1379-1387 (2013).

17. Borgia F, Goodman SG, Halvorsen S et al. Early routine percutaneous coronary intervention after fibrinolysis vs. standard therapy in ST-segment elevation myocardial infarction: a meta-analysis. Eur. Heart J. 31(17), 2156-2169 (2010). 
